\title{
Amygdala Gene Expression Correlates of Social Behavior in Monkeys Experiencing Maternal Separation
}

\author{
Michael J. Sabatini, ${ }^{1,3}$ Philip Ebert, ${ }^{7,8}$ David A. Lewis, ${ }^{1,2}$ Pat Levitt, ${ }^{1,7,8}$ Judy L. Cameron, ${ }^{1,4,5}$ and Károly Mirnics ${ }^{1,6,8}$ \\ Departments of ${ }^{1}$ Psychiatry, ${ }^{2}$ Neuroscience, and ${ }^{3}$ Neurobiology, University of Pittsburgh, Pittsburgh, Pennsylvania $15260,{ }^{4}$ Departments of Physiology and \\ Pharmacology and Behavioral Neuroscience, Oregon Health \& Science University, Portland, Oregon 97239-3098, ${ }^{5}$ Oregon National Primate Research \\ Center, Beaverton, Oregon 97006-3448, and Departments of ${ }^{6}$ Psychiatry and ${ }^{7}$ Pharmacology and ${ }^{8}$ Vanderbilt Kennedy Center for Human Development, \\ Vanderbilt University, Nashville, Tennessee 37203
}

Children exposed to early parental loss from death or separation carry a greater risk for developing future psychiatric illnesses, such as major depression and anxiety. Monkeys experiencing maternal separation at 1 week of age show fewer social behaviors and an increase in self-comforting behaviors (e.g., thumb sucking) over development, whereas in contrast, monkeys experiencing maternal separation at 1 month of age show increased seeking of social comfort later in life. We sought to identify neural systems that may underlie these stress-induced behavioral changes by examining changes in mRNA content in amygdala tissue collected from 1 week separated, 1 month separated, and maternally reared infants at 3 months of age. mRNA from the right medial temporal lobe, primarily the amygdala, was analyzed using Affymetrix U133A 2.0 arrays. One gene, guanylate cyclase $1 \alpha 3$ (GUCY1A3), showed differential expression between the 1 week and maternally reared groups and the 1 week and 1 month groups; these changes were confirmed by in situ hybridization. The expression of this gene was positively correlated with acute social-comforting behavior $(r=0.923 ; p=0.001)$ and longer-term close social behavior $(r=0.708 ; p=0.015)$ and negatively correlated with self-comforting behaviors $(r=-0.88 ; p<0.001)$. Additional in situ hybridization studies of GUCY1A3 in normal monkeys showed that this gene is expressed at adult levels by 1 week of age and that its expression is greater in the amygdala than all other brain areas examined. We conclude that GUCY1A3 may contribute to the altered behavioral phenotypes that are differentially displayed depending on the age at which macaque infants experience an early-life stress.

Key words: DNA microarray; gene expression; amygdala; maternal separation; in situ hybridization; behavior; rhesus macaque; guanylate cyclase

\section{Introduction}

Primates exposed to early-life social stress develop long-lasting behavioral changes that depend on the exact nature and timing of the stressor. In children, early parental loss from death or separation engenders an increased risk for developing future psychiatric illnesses, such as major depression and anxiety (Mireault and Bond, 1992; Agid et al., 1999). For example, Romanian orphans who received little social support before age three and one-half displayed increased autistic-like behaviors (Rutter et al., 1999) and indiscriminate social behaviors (Chisholm, 1998; O'Connor and Rutter, 2000; Rutter and O'Connor, 2004). In

\footnotetext{
Received Nov. 1, 2006; revised Jan. 3, 2007; accepted Feb. 12, 2007.

This work was supported by National Institute of Mental Health (NIMH) Grant MH051234 (D.A.L.), Vanderbilt Kennedy Center-Developmental Disabilities Research Center Grant P30 HD15052 (P.L.), the John D. and Catherine T. MacArthur Foundation Network on Early Experience and Brain Development (J.L.C.), NIMH Grants P01 MH41712 (J.L.C.), R01 MH067234 (K.M.), and 2 P50 MH45156, Conte Center for the Neuroscience of Mental Disorders Project 2 (K.M.), and NIMH Grant K02 MH070786 (K.M.). This publication is based on the doctoral thesis of M.J.S. (2005; University of Pittsburgh, Pittsburgh, PA). We thank Dr. Dominique Arion for valuable comments on this manuscript We also thank Katherine C. Douglass, Annie Bedison, and Melissa Macioce for superb technical assistance with the experiments. The expert technical assistance in rearing infant monkeys by Sally Kuhn, Sarah Acosta, Nathan Rockcastle, Erika Bauer, Denise Colosimo, Henry Lange, and Jennifer Dry-Henich was greatly appreciated.

Correspondence should be addressed to Karoly Mirnics, Department of Psychiatry, Vanderbilt University, 8130A MRB III, 465 21st Avenue South, Nashville, TN 37203. E-mail: karoly.mirnics@vanderbilt.edu.

DOI:10.1523/JNEUROSCI.4765-06.2007

Copyright (C) 2007 Society for Neuroscience $\quad 0270-6474 / 07 / 273295-10 \$ 15.00 / 0$
}

monkeys, early-life disruptions of the maternal-infant bond (e.g., maternal separation) lead to later life changes in social behaviors and increased depressive and anxious behaviors (Harlow and Zimmermann, 1959; Harlow and Suomi, 1971; Suomi et al., 1975). Specifically, monkeys whose mothers are removed from the stable social group at 1 week of age initially show increases in contact-comforting behaviors and decreases in social-contact behaviors (Cameron, 2001). They later develop increases in selfcomforting behaviors, increases in anxious behaviors, and decreases in social behaviors when in a social environment. In contrast, monkeys whose mothers are removed at 1 month of age initially show increases in social-comforting behaviors and later develop increases in social behaviors and increases in anxious behaviors (Cameron, 2001; McCormick et al., 2005). Given that this 3 week difference in the timing of the maternal-infant bond disruption produces these behavioral extremes, this paradigm is ideally suited for examinations of the brain substrate associated with the development of social and anxious behaviors.

In primates, a clear role for the amygdala in social, anxious, and depressive behaviors has been established (Brothers, 1990; Drevets, 1999; Baron-Cohen et al., 2000; Emery et al., 2001; Drevets et al., 2002; Amaral, 2003; Bauman et al., 2004; Etkin et al., 2004; Lorberbaum et al., 2004). Although the amygdala has been repeatedly implicated in behaviors that are related to early-life 
stress, no studies have examined the molecular changes that result from maternal separation in primates. We elected to examine this contribution using a "genes to behavior" approach (Nelson et al., 2002) beginning with DNA microarray gene expression profiling. Our follow-up studies focused on one gene, guanylate cyclase $1 \alpha$ 3 (GUCY1A3), intimately associated with the timing of the early-life social stress. The product of this gene is the human analog of the rat guanylate cyclase $1 \alpha 1$ (Zabel et al., 1998), an element of the nitric oxide (NO) signaling cascade (Russwurm and Koesling, 2004), and expressed in limbic structures of the prenatal and postnatal rodent brain (Furuyama et al., 1993; Smigrodzki and Levitt, 1996). We show that (1) mRNA expression of GUCY1A3 in the infant monkey's amygdala is regulated by the timing of maternal removal from the stable social group; (2) in maternally reared control monkeys, the adult levels of GUCY1A3 mRNA expression are in place by 1 week of age; (3) the amygdala has higher levels of GUCY1A3 compared with cortex, cerebellum, and basal ganglia; and (4) GUCY1A3 expression is strongly correlated with specific aspects of social behavior that are altered by the experience of early maternal separation.

\section{Materials and Methods}

All experiments were reviewed and approved by the Animal Care and Use Committee of the University of Pittsburgh.

\section{Maternal separation paradigm}

A total of 12 female rhesus monkeys born in the breeding colony at the University of Pittsburgh Primate Research Laboratory were used for these studies. At birth, each monkey was arbitrarily selected to enter the "1 week separated," "1 month separated," or "control" experimental groups ( $n=4$ per group). All monkeys in this study spent the first week of life in a single cage with their mother. During this initial period, monkeys were housed in cages in temperature-controlled $\left(24 \pm 2{ }^{\circ} \mathrm{C}\right)$ communal rooms with artificial lighting from 7:00 A.M. to 7:00 P.M., Purina Monkey Chow was provided once daily (number 5045; Ralston Purina, St. Louis, MO), and water was available ad libitum. After the first week of life, the animals were handled differently depending on experimental group assignment. At 1 week of age, monkeys designated as 1 week separated were removed from their mothers and placed in a single cage immediately adjacent to a group-rearing pen that they would ultimately join. After a 5-7 d period of learning to bottle feed (ad libitum Similac with Iron baby formula; Abbott Laboratories, Columbus $\mathrm{OH}$ ), these monkeys were introduced into the group-rearing environment. One week separated monkeys spent weeks $3-12$ in the group-rearing environment. Conversely, at 1 week of age, monkeys designated as 1 month separated were introduced into a group-rearing pen with their mothers. One month separated monkeys spent weeks 2-4 in the grouprearing environment with their mothers. At 4 weeks of age, 1 month separated monkeys' mothers were removed from the group-rearing environment, and the infants were placed in the single cage positioned identically as described for the 1 week separated group. After a 5-7 d period of learning to bottle-feed, these monkeys were reintroduced into the group-rearing environment. One month separated infants again spent weeks $6-12$ in the group-rearing environment. Monkeys from both separation groups were provided a soft-cotton stuffed toy when they were in the single cage learning to bottle feed. This toy could be held to provide contact comfort. Control monkeys were introduced into the group-rearing pen at 1 week of age with their mother, and they remained there with their mother for the study's entirety, weeks $2-12$. The group- rearing pen consisted of 4-5 monkeys of varying ages, with no monkey more dominant than the adult female mother (when present). An adolescent female was always present in the social rearing groups because they have been shown to be particularly attentive to infants. The grouprearing pen, measuring $14 \times 11 \times 12$ feet, was cement block construction with a several inch bed of wood shavings on the floor, four perches at various heights, and a chain-link penfront. A "nursery chamber", measuring $2 \times 2 \times 3$ feet, was placed in each pen and accessible only to infants. Infants had access to bottles of standard human artificial formula that were replaced three times each day. Other monkeys were fed one meal per day between 9:00 and 10:00 A.M. of Purina high-protein monkey chow supplemented several times a week with fresh fruit and seeds strewn in the bedding to encourage foraging. Water was available ad libitum. Artificial lighting in the hallways was on from 7:00 A.M. to 7:00 P.M. each day, and sky lights above each pen admitted natural light. Temperature was maintained at $24 \pm 2^{\circ} \mathrm{C}$. The infant monkeys were weighed weekly, and all animals, regardless of maternal separation status, reported a comparable weight gain over time (supplemental material 1, available at www.jneurosci.org).

\section{Behavioral assays}

Behavioral assessments were initially made at the time of separation and again during the third month of life and will be described briefly below.

Acute separation behaviors. At the time of maternal separation, each monkey (four 1 week separated and four 1 month separated) was videotaped in the single nursery cage setting for $10 \mathrm{~min}$ per focal session. This occurred two to four times per day for the first $3 \mathrm{~d}$ after maternal separation, and every other day thereafter, until the infant was transferred to the group-rearing pen. All behaviors displayed by the focal monkeys in the acute separation cage (Table 1) were scored via videotape analysis with the aid of behavioral observation software (Observer Video Pro, version 5.0; Noldus Information Technology, Wageningen, The Netherlands). Although blinding was impossible because the group differences were distinct, bias was unlikely because (1) the tapes are observed in a frame-by-frame analysis in slow motion, and (2) behaviors are rigorously defined. All tapes were scored by a single rater with intrarater reliability maintained at $90 \%$ or better. For each session, the total time spent engaging in each behavior was compiled. For most behaviors, the total time for each focal session was adjusted for the time spent in passive sit, a behavior that included, and was not distinguishable from sleeping. For each monkey, the duration data were averaged over all of the focal sessions (9-11 sessions per animal) to obtain a single mean measure for each behavior. These values were then used to compute the mean and SD for the duration of each behavior in each group of experimental monkeys.

Three month social group behavior. Each focal monkey (four per group in one week separated, 1 month separated, and control groups) was videotaped in its group-rearing environment twice per week for $15 \mathrm{~min}$. 
Table 2. Group-rearing pen behaviors

\begin{tabular}{|c|c|c|}
\hline Behavior & Description & Category \\
\hline Ventral contact & Initiate or receive a face-to-face hold with a pen mate & Social-comforting \\
\hline Huddle & Sitting with torso in contact with a pen mate (but not a face-to-face hold) & Social-comforting \\
\hline Touch & Receive or initiate contact with pen mate, not defined as ventral, huddle, or groom & Social-comforting \\
\hline Groom & Picking or brushing a penmates hair or skin & Social-comforting \\
\hline Proximity & Sitting within an arm's length of a pen mate but not touching & Social-comforting \\
\hline Social play & Initiate or receive play with a pen mate & Social-comforting \\
\hline Atypical social & Thumb-sucking or toe-sucking while in contact with or proximity to a penmate & Social-comforting \\
\hline Self-comforting & Thumb-sucking or toe-sucking & Self-comforting / Nonsocial \\
\hline Snuggly & Contact with snuggly toy & Self-comforting / nonsocial \\
\hline Object Play & Manipulating cage or cage objects & Nonsocial \\
\hline Stationary & Stationary & Nonsocial \\
\hline Locomote & Walking, running, and pacing in the pen or on a perch & Nonsocial \\
\hline Eat & Foraging for food, putting food in mouth, drinking & Nonsocial \\
\hline Fence Biting & Biting or chewing cage at pen front & Nonsocial \\
\hline Scratch & Scratch oneself & Nonsocial \\
\hline Nursery & Inside nursery chamber & Nonsocial \\
\hline Agitation & Rapid head movements & Nonsocial \\
\hline Aggression & Receive or initiate threatening gesture or aggressive contact & Other \\
\hline
\end{tabular}

Summary of behaviors measured in the group rearing pen. Behaviors are inclusive but not necessarily mutually exclusive. Category delineates behaviors associated with social-comforting or self-comforting.

Scoring was accomplished by a single observer via videotape analysis with the aid of behavioral observation software (Observer Video Pro), as described in the acute behavior section previously. Each $15 \mathrm{~min}$, individual focal session was completely analyzed for duration and frequency of the behaviors displayed in the group-rearing pen as listed in Table 2. Each behavior was adjusted for the time spent in the nursery chamber. This chamber did not allow an unobstructed view of the monkey, and therefore specific behaviors could not be reliably scored. For each monkey, the eight focal sessions (2 per week) during weeks 9-12 were used for analysis. Previous studies in our laboratory suggest that this number of sessions produces a reliable measure of stable behavior at this age (Cameron, 2001).

\section{Medial temporal lobe tissue collection}

After the behavioral analyses at 12 weeks of age, brain tissue was collected from each experimental monkey for molecular studies. Monkeys were deeply anesthetized with ketamine/pentobarbitol, quickly decapitated, and the entire brain was removed from the skull. The brain was then hemisected by a midline sagittal cut. The right hemisphere was cut into $\sim 5$-mm-thick coronal blocks, flash frozen in isopentane over dry ice, and stored at $-80^{\circ} \mathrm{C}$ until cryostat sectioning as described previously (Volk et al., 2000). Immediately before cryostat sectioning, the amygdala and periamygdalar cortex were dissected from the coronal blocks using a cut parallel to the midline between the amygdala lateral nucleus boundary and the invagination of the superior temporal sulcus, and a perpendicular cut just superior to the optic tract. The dissected blocks were then sectioned using a cryostat at $20 \mu \mathrm{m}$ throughout the region containing the amygdala. From each set of 20 sections $(400 \mu \mathrm{m}), 11$ sections were thawmounted onto Superfrost Plus micro slides (VWR Scientific, West Chester, PA) and nine sections were collected into TRIzol reagent (Invitrogen, Carlsbad, CA) for total RNA isolation, vortexed for $1 \mathrm{~min}$, and frozen at $-80^{\circ} \mathrm{C}$. One thaw-mounted section in each set of 20 was stained for Nissl substance using thionin. The identification of two distinct landmarks, the most rostral aspect of the lateral ventricle and the glomerulus of the amygdala paralaminar nucleus allowed the mapping of each medial temporal lobe onto one another, ensuring that the same region of the medial temporal lobe was used for each subject. The set of 20 sections nearest the most rostral boundary of the lateral ventricle was used for both microarray and in situ hybridization experiments.

\section{Microarray experiments}

Total RNA was isolated according to the manufacturer's instructions including all optional steps. The isolations yielded between 10 and $15 \mu \mathrm{g}$ of total RNA from each subject. Each total RNA sample was assessed for purity using the absorption ratio between 260 and $280 \mathrm{~nm}$ in an ultraviolet spectrophotometer and quality using the 28 to $18 \mathrm{~s}$ ribosomal sub- unit ratio from the electropherogram of the BioAnalyzer (Agilent, Palo Alto, CA). Samples were considered appropriate for microarrays with an A260/A280 $\geq 1.8$, and a $28 \mathrm{~s} / 18 \mathrm{~s} \geq 1.4$. Reverse transcription, in vitro transcription amplification, and fragmentation were performed according to the manufacturer's instructions (Affymetrix, Santa Clara, CA). Using the Affymetrix hybridization station, each sample was hybridized onto an Affymetrix Human Genome U133A 2.0 Array, which contained $\sim 22,000$ probesets that characterize $>18,500$ unique transcripts. To avoid microarray batch variation, only microarrays from a single lot were used. Segmentation of scanned microarray images was performed by Microarray Analysis Suite 5.0. Determination of expression levels and scaling were performed using Robust Multiarray Average (RMA) (Irizarry et al., 2003). Microarrays were considered for use only if the average $5^{\prime}: 3^{\prime}$ ratio for GAPDH and actin did not exceed 1:1.2.

\section{GUCY1A3 in situ hybridization}

In situ hybridization studies were performed according to methods described previously (Mirnics et al., 2001). Briefly, gene-specific 20-mer primers were designed using the human soluble GUCY1A3 sequence, GenBank accession number NM_000856. Forward primer was TGTACACTCGCTTCGACCAG; reverse primer was TCCAGAAAATGGCAGATTCC. Using these primers a $448 \mathrm{bp}$ amplicon was generated by PCR amplification using monkey medial temporal lobe cDNA as a template. The resulting fragment was T/A cloned into the Acceptor pstBlue-1 vector (Novagen, San Diego, CA). The GUCY1A3 clone was confirmed by sequencing. Dual opposed promoters allowed efficient in vitro transcription of both the forward and reverse products. Labeled antisense RNA probes were generated using in vitro transcription in the presence of ${ }^{35}$ S-labeled CTP (GE Healthcare, Piscataway, NJ). An amount of probe yielding 2 million dpm was hybridized overnight onto 20 - $\mu$ m-thick, thaw-mounted tissue sections that were lightly fixed in $4 \%$ paraformaldehyde, dehydrated in graded alcohols, and delipidated in chloroform. After a series of stringent washes and RNase treatment to limit nonspecific binding, slides were dried and exposed to x-ray film [Kodak (Rochester, NY) MR Gold] for $2 \mathrm{~d}$. Known ${ }^{14} \mathrm{C}$ standard slides were included for quantitative analysis. A sense RNA probe was treated in parallel as a specificity control, and no signal was present after $2 \mathrm{~d}$ of exposure. Radiographic films were scanned at $800 \mathrm{dpi}$ and imported into Scion (Frederick, MD) Image for optical density measurements. The slides were subsequently dipped in photo emulsion. Dipped slides were exposed for 7 d, developed with Kodak D-19 developer, fixed with 30\% sodium thiosulfate, and visualized under light- and dark-field microscopy.

GUCY1A3 mRNA expression in normal postnatal development For the developmental studies, we chose five postnatal time points: 1, 4, 12, 63, and 176 weeks. Four monkeys each were analyzed at 1 and 4 
weeks, six monkeys at 12 weeks, and three monkeys were analyzed at 63 and 176 weeks. None of the monkeys used for these experiments were subjected to early-life social stress. Some monkeys $(n=6)$ had undergone previous brain biopsy of the prefrontal cortex (Gonzalez-Burgos et al., 2000, 2005). All of the monkeys in the 1 week and 1 month groups and four of six monkeys in the 12 week group were raised as described above in group-rearing pens with three to five other monkeys of varying ages. Monkeys from the 63 week and 176 week groups, and two of six monkeys from the 12 week group were reared with their mothers in single cages in communal rooms. In the single-cage reared monkeys, weaning occurred at 6 months of age when applicable. Right amygdala collection and sectioning was identical to the monkeys in the maternal separation paradigm. In situ hybridization was accomplished by the same method and used the same template for probe production.

\section{GUCY1A3 normal regional $m R N A$ expression}

Two monkeys, ages 4 months and 6 months, were used for these studies. Neither monkey was subjected to early-life social stress. Coronal sections from the right hemisphere from four rostrocaudal levels and sagittal sections from the cerebellum were collected in an analogous manner to those described in the medial temporal lobe collection. In situ hybridization was accomplished by the same method and used the same template for probe production.

\section{Data analysis}

Microarray. RMA analysis yielded normalized, $\log 2$-transformed expression levels for each of the $>18,500$ transcripts probed. We considered a gene present when its RMA-scaled expression level was $>6.49$. Twotailed Student's $t$ tests were performed between experimental groups, and a transcript was considered differentially regulated between groups when (1) the difference between the group averages was at least 1.2-fold, and (2) the $t$ test $p$ value was $\leq 0.01$. This present-absent cutoff was set based on previous success of verifying expression levels of genes at or above this level by either real-time PCR or in situ hybridization. False discovery assessment was performed using SAM (Significance Analysis of Microarray) in $\mathrm{R}$ using 100 random permutations (Storey and Tibshirani, 2003).

In situ hybridization. The emission of $\beta$ particles from specifically bound probe was determined by measuring the mean gray level around the extent of the amygdala on the radiographic film images using the polygonal selection tool in Scion Image. The observer was blinded to experimental group. Known ${ }^{14} \mathrm{C}$ standards were included in each exposure to ensure that the maximum gray level measured within the amygdala was within the linear range of the radiographic film and to calibrate the gene expression data. When quantitative measurement was indicated, the background gray level for the film surrounding the section was subtracted from each measurement. The background-subtracted gray level measurements were transformed into uncalibrated optical densities (ODs) using the following transformation: Uncalibrated OD $=\log 10$ [255/(255 - Gray Value)]. The uncalibrated OD measurements were then calibrated to known quantities of ${ }^{14} \mathrm{C}$. The expression levels from three independent sections from each experimental animal were averaged together. Expression levels are reported as microcuries per gram of ${ }^{14} \mathrm{C}$. The Kolmogorov-Smirnoff test was used to test for normality. A one-way ANOVA was performed, and after a Levene's test to ensure homoscedasity, least significant difference post hoc tests were performed. Significance was defined at $p \leq 0.05$.

Gene-behavior correlations. Pearson correlations were used to determine $r$ values and a $t$-distribution table with $n-2$ degrees of freedom was used to determine the associated two-tailed $p$ values. $r$ values were considered significant when the associated $p$ value was $\leq 0.05$. We report both the $r$ value and its associated $p$ value in the results.

\section{Results \\ Maternal separation did not affect food intake and weight gain}

To ensure that the experimental protocol did not affect general growth and development, each separated monkey was weighed weekly throughout the study and compared with control monkeys at the time they were killed. Developmental trajectories were similar among all animals, and at the time the animals were killed, there were no significant differences between any of the experimental groups (ANOVA, $F=0.284, p=0.76$ ) (supplemental material 1 , available at www.jneurosci.org).

\section{Amygdala transcriptome changes resulting from early maternal separation}

Microarray studies were used to gain an unbiased survey of gene expression differences in the medial temporal lobe, predominantly the amygdala, between monkeys whose mothers were removed from their social group at different developmental time points. Based on previous work in our laboratory and empirically on this dataset (e.g., known low-level expression genes), we defined a gene as present if its RMA normalized gene expression value was at least 6.49 when averaged across all subjects. Of the $>18,500$ unique transcripts measured, 8405 were called present according to this criterion. From these genes, we designated a gene as differentially regulated between groups if in at least one of the four comparisons (control vs 1 week, control vs 1 month, 1 week vs 1 month, and control vs 1 month plus 1 week) (1) the difference of the group averaged RMA expression levels was at least 0.263 (1.2-fold difference), and (2) the Student's $t$ test $p$ value was $<0.01$. Using these criteria, 19 genes were differentially regulated in the control versus 1 week comparison (mean Q value, 0.15$), 22$ genes were differentially regulated in the control versus 1 month comparison (mean Q value, 0.19), and only four genes were differentially regulated in the 1 week versus 1 month comparison (mean Q value, 0.68) (Tables 3, 4, 5). One gene was shared between the control versus 1 week and control versus 1 month comparisons [UBE3B (ubiquitin protein ligase E3B)]. Similarly, one gene was shared between the control versus 1 week and 1 month versus 1 week comparisons (GUCY1A3). In addition, when the 1 week separated and the 1 month separated monkeys were combined into a single group and compared with the maternally raised controls, 47 genes reported differential expression (mean Q value, 0.09) (supplemental material 2, available at www.jneurosci.org). Of these, 39 genes were underexpressed in the separated monkeys, whereas only eight reported increased transcript levels. In an unsupervised two-way (genes and animals) hierarchical clustering of the $\log 2$ expression values, these 47 transcripts clearly segregated the animals into maternally raised and maternally separated clusters (supplemental material 3, available at www.jneurosci.org).

Regardless of the statistical significance, and given the heterogeneous nature of the tissue samples and arrays (monkey RNA hybridized to human microarray chips), we felt that each gene should be validated by an alternate method; in situ hybridization studies were well suited for this task because they additionally provide anatomical detail.

\section{GUCY1A3 is decreased in the amygdala after early social bond disruption}

GUCY1A3 became the primary focus of additional studies because (1) it was the only gene found to be differentially regulated in the 1 week versus 1 month comparison, (2) it was the most changed gene by microarray in the control versus 1 week comparison, (3) the microarray data suggested high expression in the amygdala, and (4) its participation in the nitric oxide signaling cascade suggested that differences could be potentially meaningful from the standpoint of cell signaling and behavior. In the microarray studies, GUCY1A3 expression in the rostral medial temporal lobe revealed a nonsignificant decrease of $9.2 \%$ in the 1 month separated monkeys compared with maternally reared 
Table 3. Gene expression differences according to separation group: separated at 1 week versus maternally raised

\begin{tabular}{|c|c|c|c|c|c|c|}
\hline Probe set & Gene name & Symbol & Accession & UniGene & pVal & ALR \\
\hline 221942_s_at & Guanylate cyclase 1, soluble, $\alpha 3$ & GUCY1A3 & Al719730 & Hs. 24258 & 0.00360 & -0.49 \\
\hline 214315_x_at & Calreticulin & CALR & Al348935 & Hs.515162 & 0.00330 & -0.46 \\
\hline 210649_s_at & AT-rich interactive domain $1 \mathrm{~A}$ & ARID1A & AF231056 & Hs.468972 & 0.00618 & -0.34 \\
\hline 204957_at & Origin recognition complex, subunit 5 & ORC5L & NM_002553 & Hs.432948 & 0.00747 & -0.32 \\
\hline 210040_at & Solute carrier family 12, member 5 & SLC12A5 & AF208159 & Hs.21413 & 0.00417 & -0.32 \\
\hline 212403_at & Ubiquitin protein ligase E3B & UBE3B & Al749193 & Hs.374067 & 0.00816 & -0.32 \\
\hline 208906_at & Congenital lipodystrophy 2 (seipin) & $\mathrm{BSCL} 2$ & BC004911 & Hs.533709 & 0.00703 & -0.30 \\
\hline 209003_at & Solute carrier family 25, member 11 & SLC25A11 & AF070548 & Hs.592122 & 0.00193 & -0.30 \\
\hline 209117_at & WW domain-binding protein 2 & WBP2 & U79458 & Hs.514489 & 0.00327 & -0.30 \\
\hline 217221_x_at & RNA-binding motif protein 10 & RBM10 & AL137421 & Hs.401509 & 0.00639 & -0.29 \\
\hline 220166_at & Cyclin M1 & CNNM1 & NM_020348 & Hs.274579 & 0.00557 & -0.29 \\
\hline 202682_s_at & Ubiquitin-specific peptidase 4 & USP4 & NM_003363 & Hs.631919 & 0.00830 & -0.28 \\
\hline 218942_at & PI-4-phosphate 5-kinase 2, $\gamma$ & PIP5K2C & NM_024779 & Hs. 144502 & 0.00893 & -0.28 \\
\hline 201550_x_at & Actin, $\gamma 1$ & ACTG1 & NM_001614 & Hs.514581 & 0.00039 & -0.27 \\
\hline 209352_s_at & SIN3 homolog B & SIN3B & AB014600 & Hs.13999 & 0.00553 & -0.27 \\
\hline 212503_s_at & DIP2 disco-interacting protein $2 C$ & DIP2C & N22859 & Hs.432397 & 0.00442 & -0.27 \\
\hline 205208_at & Aldehyde dehydrogenase 1, L1 & ALDH1L1 & NM_012190 & Hs.434435 & 0.00958 & -0.27 \\
\hline 217197_x_at & Hypothetical gene CG018 & CG018 & AL049785 & Hs. 161220 & 0.00661 & 0.33 \\
\hline 206057_x_at & Sialophorin (leukosialin, CD43) & SPN & NM_003123 & Hs.632188 & 0.00883 & 0.35 \\
\hline
\end{tabular}

Summary of gene expression changes in each of the three possible comparison groups. Selection criteria were as follows: (1) RMA scaled expression level $>6.49$, (2) ALR $>0.263$ (1.2-fold change), and (3) $p<0.01$. Genes are sorted within each comparison based on the absolute value of the expression difference. ALR, Difference of the log2 RMA normalized expression level between groups.

Table 4. Gene expression differences according to separation group: separated at 1 week versus separated at 1 month

\begin{tabular}{|c|c|c|c|c|c|c|}
\hline Probe set & Gene name & Symbol & Accession & UniGene & pVal & ALR \\
\hline 221942_s_at & Guanylate cyclase 1, soluble, $\alpha 3$ & GUCY1A3 & Al719730 & Hs.24258 & 0.00766 & -0.36 \\
\hline 201167_x_at & Rho GDP dissoc inh (GDI) alpha & ARHGDIA & D13989 & Hs.159161 & 0.00026 & -0.32 \\
\hline 214219_x_at & MAP kinase kinase kinase kinase 1 & MAP4K1 & BE646618 & Hs.95424 & 0.00627 & -0.30 \\
\hline 213547_at & Cullin-associated neddylation 2 & CAND2 & AB014567 & Hs.343664 & 0.00915 & 0.28 \\
\hline
\end{tabular}

Table 5. Gene expression differences according to separation group: separated at 1 month versus maternally raised

\begin{tabular}{|c|c|c|c|c|c|c|}
\hline Probe set & Gene name & Symbol & Accession & UniGene & pVal & ALR \\
\hline 202289_s_at & Transforming, acidic coiled-coil 2 & TACC2 & NM_006997 & Hs.501252 & 0.00620 & -0.39 \\
\hline 201939_at & Polo-like kinase 2 & PLK2 & NM_006622 & Hs.398157 & 0.00985 & -0.38 \\
\hline 201340_s_at & Ectodermal-neural cortex & ENC1 & AF010314 & Hs. 104925 & 0.00813 & -0.38 \\
\hline 218623_at & HMP19 protein & HMP19 & NM_015980 & Hs. 559412 & 0.00619 & -0.36 \\
\hline 221743_at & CUG triplet repeat, RNA-binding 1 & CUGBP1 & Al472139 & Hs.632137 & 0.00141 & -0.36 \\
\hline 212119_at & Ras homolog gene family, member 0 & RHOQ & BF670447 & Hs.584872 & 0.00604 & -0.36 \\
\hline 205613_at & Synaptotagmin XVII & SYT17 & NM_016524 & Hs.258326 & 0.00482 & -0.32 \\
\hline 200083_at & Ubiquitin-specific peptidase 22 & USP22 & AA621731 & Hs.462492 & 0.00665 & -0.32 \\
\hline 40472_at & 1-Acylglycl-3-phosph 0-acyltransf 7 & AGPAT7 & AF007155 & Hs.352614 & 0.00848 & -0.30 \\
\hline 213308_at & SH3 and ankyrin repeat domains 2 & SHANK2 & BF435773 & Hs. 268726 & 0.00700 & -0.30 \\
\hline 204141_at & Tubulin, $\beta 2 \mathrm{~A}$ & TUBB2A & NM_001069 & & 0.00496 & -0.30 \\
\hline 212403_at & Ubiquitin protein ligase E3B & UBE3B & Al749193 & Hs.374067 & 0.00759 & -0.29 \\
\hline 209871_s_at & Amyloid $\beta$ precursor-binding, A2 & APBA2 & AB014719 & Hs.525718 & 0.00027 & -0.29 \\
\hline 212705_x_at & Patatin-like phospholipase 2 & PNPLA2 & BF570210 & & 0.00590 & 0.27 \\
\hline 209622_at & Serine/threonine kinase 16 & STK16 & AB020739 & Hs.153003 & 0.00030 & 0.27 \\
\hline 211162_x_at & Stearoyl-coenzyme A desaturase & SCD & AF116616 & Hs.558396 & 0.00355 & 0.29 \\
\hline 215672_s_at & KIAA0828 protein & KIAA0828 & AK025372 & Hs.600789 & 0.00260 & 0.30 \\
\hline 203167_at & TIMP metallopeptidase inhibitor 2 & TIMP2 & NM_003255 & Hs.104839 & 0.00694 & 0.31 \\
\hline 222264_at & hn ribonucleoprotein U-like 2 & HNRPUL2 & BG167570 & Hs.406377 & 0.00678 & 0.33 \\
\hline 210684_s_at & Discs large homolog 4 & DLG4 & AF028825 & Hs.463928 & 0.00669 & 0.34 \\
\hline 210457_x_at & High-mobility group AT-hook 1 & HMGA1 & AF176039 & Hs.518805 & 0.00163 & 0.37 \\
\hline 209070_s_at & Regulator of G-protein signaling 5 & RGS5 & Al183997 & Hs. 568700 & 0.00769 & 0.45 \\
\hline
\end{tabular}

controls and a significant decrease of $29.1 \%(p=0.004)$ in 1 week separated monkeys compared with controls. One week separated monkeys, when compared with 1 month separated monkeys, revealed a significant decrease of $22.0 \%(p=0.008)$ in GUCY1A3 expression (Fig. 1A).

In situ hybridization on tissue sections adjacent to those used for microarray was performed for GUCY1A3. Mean expression of GUCY1A3 was significantly different $(p<0.001)$ between maternally raised monkeys $(0.486 \pm 0.063 \mu \mathrm{Ci} / \mathrm{g}), 1$ week separated monkeys $(0.245 \pm 0.048 \mu \mathrm{Ci} / \mathrm{g})$, and 1 month separated monkeys $(0.422 \pm 0.060 \mu \mathrm{Ci} / g)$ (Fig. $1 B$ ). Post hoc tests revealed that a significant difference exists in the control versus 1 week 

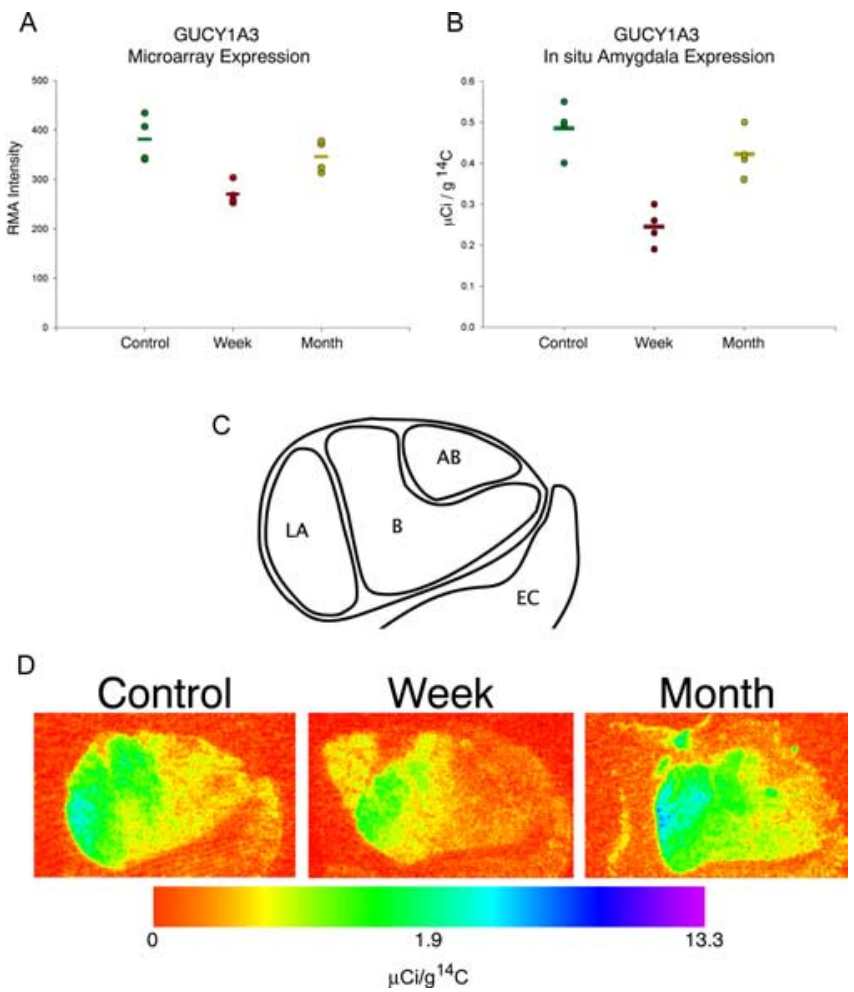

Figure 1. GUCY1A3 mRNA expression. $A, G U C Y 1 A 3$ microarray gene expression level in the rostral medial temporal lobe. Circles represent the RMA normalized expression levels from each individual monkey; bars represent group mean. One week separated monkeys show significant decreases of 29.1\% compared with controls and 22.0\% compared with 1 month separated monkeys ( $p<0.01$ ). B, Quantification of radiographs of GUCY1A3 ${ }^{35}$ S-labeled ribroprobe around the extent of the amygdala. Optical density levels were converted to known activities of ${ }^{14} \mathrm{C}$ standards and expressed in microcuries per gram of ${ }^{14} \mathrm{C}$. Circles represent the average expression levels from three sections in each individual monkey; bars represent the group mean. Data were analyzed using ANOVA followed by least significant difference post hoc tests. Significant differences existed in comparisons of 1 week separated versus control monkeys and 1 week separated versus 1 month separated monkeys $(p<0.05)$. C, Graphic depiction of the region of the amygdala assessed in both the microarray and in situ hybridization studies. $L A$, Lateral nucleus of the amygdala; $B$, basal nucleus of the amygdala; $A B$, accessory basal nucleus of the amygdala; EC, entorhinal cortex. D, Representative pseudocolored image depicting regional amygdala expression in one animal per group expressed in a log scale. Each panel depicts the right amygdala at the coronal level immediately rostral to the lateral ventricle. Subnuclei evident in these panels from lateral (left) to medial (right) are as follows: lateral nucleus, basal nucleus, and accessory basal nucleus. Medial and adjacent to the amygdala is the periamygdalar cortex; inferomedial to the amygdala is the entorhinal cortex. Control, maternally raised; Week, 1 week separated; Month, 1 month separated. Color indicates the level of expression compared with ${ }^{14} \mathrm{C}$ standard slides and is expressed in microcuries per gram of ${ }^{14} \mathrm{C}$.

comparison $(p<0.001)$ and in the 1 week versus 1 month comparison $(p=0.001)$ but not in the control versus 1 month comparison $(p=0.194)$. The decrease in the 1 week separated monkeys was distributed throughout the amygdala (Fig. $1 C, D)$ but was especially prominent in the lateral and basal nuclei.

A representative field from the lateral nucleus of the amygdala from one maternally reared subject appears in Figure 2. Hybridization appears as punctuate labeling of developed silver grains over cell bodies counterstained with cresyl-violet (see solid arrow for typical appearance of an expressing cell). Silver grains were observed in large, lightly counterstained cells, presumably projection neurons, and absent from smaller more darkly counterstained cell bodies, presumably glia. This cellular pattern was identical in both separation conditions and in all of the other amygdala subnuclei (data not shown).

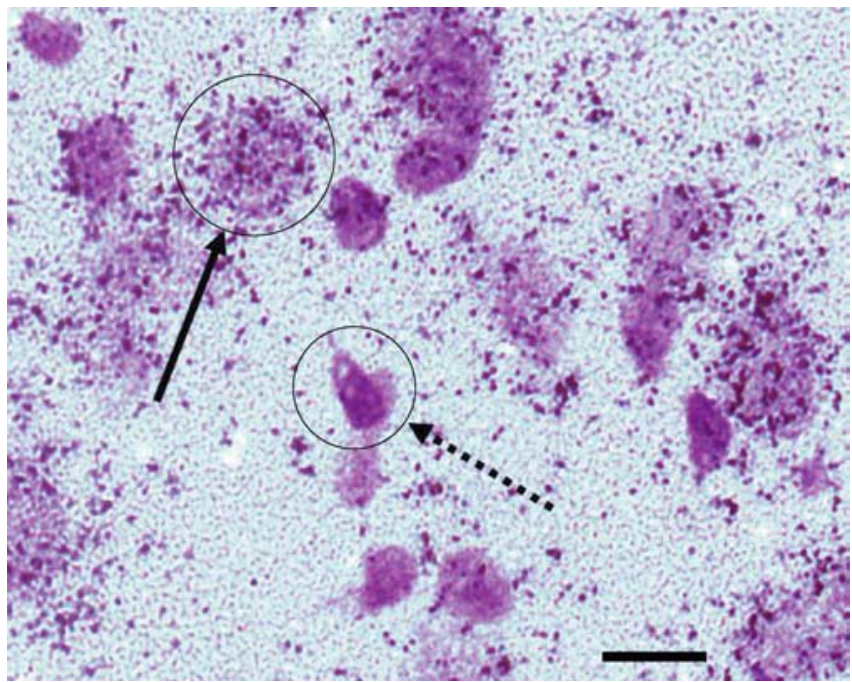

Figure 2. Cellular and regional distribution of GUCY1A3. Representative photomicrograph from the lateral nucleus of the amygdala from one maternally raised control monkey. ${ }^{35} \mathrm{~S}$ labeled riboprobes for GUCY1A3 appear as punctuate labeling of silver grains clustered around cell bodies counterstained with cresyl violet. The solid arrow depicts a typically labeled cell. The dashed arrow depicts a typical cell devoid of GUCY1A3 mRNA labeling. Scale bar, $10 \mu \mathrm{m}$.

\section{GUCY1A3 is expressed at highest levels in the amygdala}

From the limited observations of the in situ hybridization-labeled sections in the medial temporal lobe, it appeared that GUCY1A3 was most highly expressed in the amygdala. In situ hybridization label distribution revealed a subnucleus-specific expression pattern with strongest labeling in the lateral and basal nuclei (Fig. $1 C, D)$. In contrast, the accessory basal nucleus showed only moderate GUCY1A3 labeling. Interestingly, this pattern is similar to the previously reported serotonin axon density in the primate amygdala (Hatzidimitriou et al., 1999).

Because the spatial distribution of GUCY1A3 across the primate brain has not been established, we systematically surveyed the expression of GUCY1A3 in two maternally reared control monkeys. As expected, the amygdala showed the highest level of expression compared with multiple areas of the prefrontal, temporal, parietal, and occipital cortices, as well as the cerebellum, caudate nucleus, putamen, and claustrum (Fig. 3). Interestingly, GUCY1A3 was also prominent in regions surrounding the amygdala: the periamygdalar cortex, inferior temporal cortex, and parts of the insular cortex. All regions of the neocortex examined expressed GUCY1A3 in a complex, multilaminar pattern.

\section{GUCY1A3 is expressed at adult levels by 1 week of age}

The observed lower expression of GUCY1A3 transcript in the amygdala of 1 week separated animals could conceivably arise by two different mechanisms: (1) decreased expression after maternal separation or (2) a failure to increase GUCY1A3 expression levels during normal postnatal development. To discriminate between these alternatives, we examined amygdala GUCY1A3 expression levels in control monkeys at five time points between 1 and 176 weeks of age. Microscopic inspection of the anatomical distribution of GUCY1A3 labeling in the medial temporal lobe revealed a similar expression pattern in the amygdala at all ages examined, whereas the cortical, laminar expression seemed to vary with age (Fig. 4A-E). We found no evidence of significant expression level differences in the amygdala between the five studied ages (ANOVA, $F_{(4,15)}=0.937, p=0.469$ ) (Fig. $4 F$ ). These data suggest that GUCY1A3 is expressed in the amygdala at 

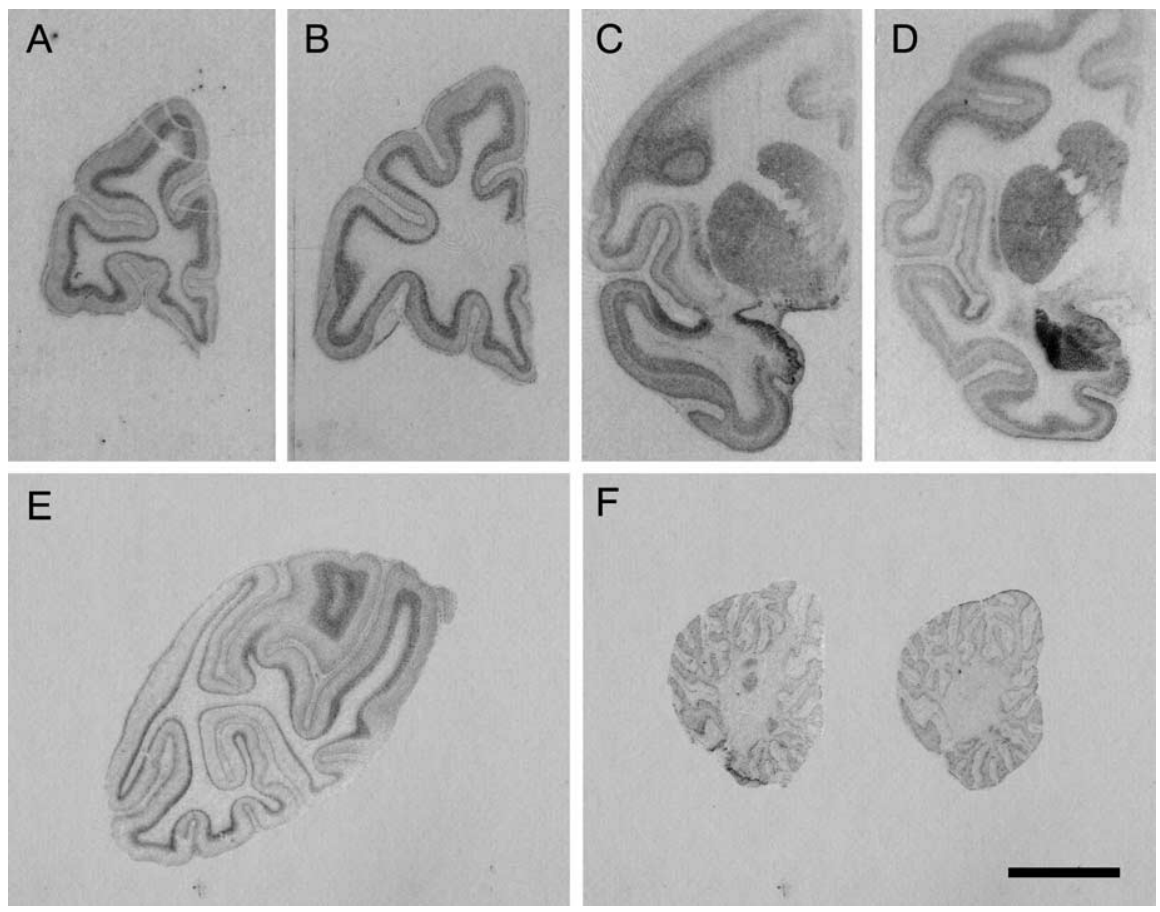

Figure 3. Regional survey of GUCY1A3 expression in the monkey brain. Representative photomicrographs in a control monkey from: multiple coronal planes through forebrain $(\boldsymbol{A}-\boldsymbol{E})$ and two sagittal planes through the cerebellum $(\boldsymbol{F})$. Labeled GUCY1A3 mRNA appears as graded levels of gray. Note the intense labeling in and around the amygdala, especially the lateral nucleus, and the periamygdalar cortex. Scale bar, $1 \mathrm{~cm}$.

or near adult levels by 1 week of age and that the differential labeling of GUCY1A3 in the 1 week separated monkeys is attributable to transcript downregulation after early stress rather than the inability to normally increase expression levels during postnatal development.

\section{GUCY1A3 expression is correlated with behavior}

The expression data of GUCY1A3 appeared to strongly correlate with the behavioral phenotypes of monkeys observed during the third month of life (Cameron, 2001). GUCY1A3 expression in 1 week separated monkeys was significantly lower than in control monkeys, and 1 month separated animals were intermediate between the two but closer to the control monkey's expression. These differences were similar to certain aspects of socioemotional behavior that were measured either acutely at the time of separation or in their stable long-term group environment. To test for a statistical correlation, behaviors that defined the groups both acutely at the time of separation (Table 1) and at 3 months of age (Table 2) were assessed as a function of GUCY1A3 expression measured by in situ hybridization. Analysis of self-comforting and social-comforting behaviors in the acute period, as well as self-comforting and typical social behaviors in the postseparation period revealed that self-comforting behavior was significantly negatively correlated with GUCY1A3 amygdala expression $(r=$ $-0.878 ; p<0.001$ ) (Fig. 5A), and typical social behavior was significantly positively correlated with GUCY1A3 amygdala expression $(r=0.708 ; p=0.015)$ (Fig. $5 B)$ in the period of postseparation rearing-group interaction. Interestingly, the 3 month expression level of GUCY1A3 was also correlated with the acute social-comforting behavior $(r=0.923$; $p=0.001)$ (Fig. $5 C$ ) but did not reach statistical significance when correlated with the acute self-comforting behavior $(r=-0.0637$; $p=0.09)$.

\section{Discussion}

In this study, we examined gene expression changes in the amygdala at 3 months of age in three groups of monkeys ( 1 week separated, 1 month separated, and maternally reared) that differed with respect to the period of early maternal rearing. DNA microarray analysis of the amygdala revealed many interesting gene expression differences (including transcripts of many genes involved in intracellular signaling); we concentrated our follow-up studies on GUCY1A3 because (1) it was the most changed gene between the 1 week separated and maternally reared groups; (2) it was one of the few genes whose expression differed between the 1 week separated and 1 month separated groups; (3) it was one of the highest expressors of the genes identified in the amygdala; and (4) it is part of the NO signaling cascade. The subsequent quantitative in situ hybridization studies confirmed a robust downregulation of GUCY1A3 mRNA throughout the amygdala in the 1 week separated group compared with both the maternally reared and 1 month separated groups. This downregulation was most apparent in the lateral and basal nuclei in which this gene is heavily expressed in maternally reared controls.

Previous studies have described the behavioral differences after this maternal separation paradigm (Cameron, 2001; McCormick et al., 2005). In terms of those behavioral studies, the biggest difference between groups existed between the maternally reared controls and the 1 week separated monkeys. In these studies, key differences were noted in the types of comforting behaviors (selfcomforting vs social-comforting) and the amount of social interaction behavior that occurred in the various rearing groups. In this report, we have shown that the expression of GUCY1A3 is significantly correlated with two behaviors that were measured in the social rearing-group environment during the month preceding the collection of brain tissue: self-comforting behaviors (thumb-sucking and toe-sucking), and typical social behaviors. Increased self-comforting behavior in monkeys has been previously implicated as a sign of anxiety (Harlow and Suomi, 1974). Interestingly, GUCY1A3 expression at 3 months of age was also tightly correlated with the tendency to seek social comfort at the time of separation. The latter correlation raises the possibility that after a substantial stressor, behavior in the acute stress period may predict which monkeys will go on to have the largest decreases in GUCY1A3 expression later in life.

The amygdala, in addition to being involved in fear and anxiety, plays a role in feeding behavior and reward processing (Petrovich et al., 2005; Paton et al., 2006). Thus, it is possible that altered feeding behavior may give rise to gene expression changes in the amygdala, potentially confounding our dataset. However, multiple lines of evidence suggest that the observed gene expression changes are not related to nutrition. First, food intake, as judged by weight gain, is the same among all animals studied regardless of separation condition. Second, basic diet components are also similar among all animals (breast milk vs baby 
formula). Although there may be an increased tendency for the separated animals to eat the monkey chow at an earlier age, the bulk of the calories are still coming from the formula through the time period studied (3 months). Third, gene expression studies of caloric restriction in other brain areas result in gene expression changes that do not overlap with the data obtained in our study (Weindruch et al., 2002). Finally and most importantly, reports from other mammals support the notion that the NO signaling pathway, in which GUCY1A3 participates, directly modulates behaviors relevant to this study. For example, intra-amygdala injection of an inhibitor of NO synthase results in increased anxiety in rats (Monzon et al., 2001), knock-out of the major downstream target of cGMP, cGKII, leads to increased anxiety-like behavior in light/dark test and elevated maze test (Werner et al., 2004), and systemic administration of L-NAME or 7-nitroindazole, both NO synthase inhibitors, reduces anxiety in the elevated plus maze (Volke et al., 1995, 1997).

Exactly how the decreased GUCY1A3 expression translates into behavioral changes requires more study, but many aspects of the function of this gene have been previously worked out. It is known that neurons of the monkey basolateral amygdala produce nitric oxide (McDonald and Augustine, 1993; McDonald et al., 1993). GUCY1A3 acts as the soluble receptor for $\mathrm{NO}$ in a variety of brain tissues, in which it responds to NO binding by stimulating the production of cGMP (Ahern et al., 2002). It is thought to mediate its actions via downstream protein kinase $\mathrm{G}$, cyclic-nucleotide-gated channels, and cGMP-dependent phosphodiesterases. It has been suggested that NO generated postsynaptically by $\mathrm{Ca}^{2+}$-calmodulin-dependent $\mathrm{NO}$ synthase may act as a retrograde messenger and bind to presynaptic guanylate cyclase to produce cGMP-dependent downstream effects, which strengthen the synaptic connection between coordinately firing cells (Hawkins et al., 1998; Son et al., 1998). At the level of systems physiology, one critical function of guanylate cyclase is the facilitation of long-term potentiation in the amygdala (Chien et al., 2003). In rat amygdala slice recordings, stimulation of guanylate cyclase by $\mathrm{YC}-1$ leads to enhanced and enduring potentiation of the corticoamygdala pathway, and this potentiation could be eliminated by concomitant inhibition of NO synthase by L-NAME (Chien et al., 2003). Other studies have shown in vivo that inhibition of $\mathrm{NO}$ synthase impairs memory in rats as assessed by the Morris water maze (Holscher et al., 1996), and recently, Chien et al. (2005) have tested YC-1 in vivo and found improvements in Morris water maze performance. Thus, if decreased long-term potentiation in the amygdala is responsible for the behavioral deficits in early separated monkeys, drug treatment with
YC-1 may be a promising avenue for future therapeutic intervention.

It is important that our findings are interpreted in the context of the developing brain, and the observed gene expression changes may be a result of altered development and connectivity of the amygdala. The NO system is known to modulate neurite outgrowth in a complex manner. Depending on the system studied, NO-releasing compounds have been shown to either induce growth cone collapse (Hess et al., 1993; He et al., 2002) or increase neurite extension (Hindley et al., 1997; Rialas et al., 2000). This is especially relevant to the interpretation of the current study because some aspects of amygdala development continue throughout early postnatal life. Although the data on the development of the primate amygdala is limited, it appears that there is a significant postnatal reshaping of amygdala connectivity (Webster et al., 1991), and amygdala-temporal cortex connections may not be fully functional in monkeys until 3 months of age (Rodman and Consuelos, 1994). Thus, qualitative and quantitative connectivity studies of our experimental model may reveal structural 

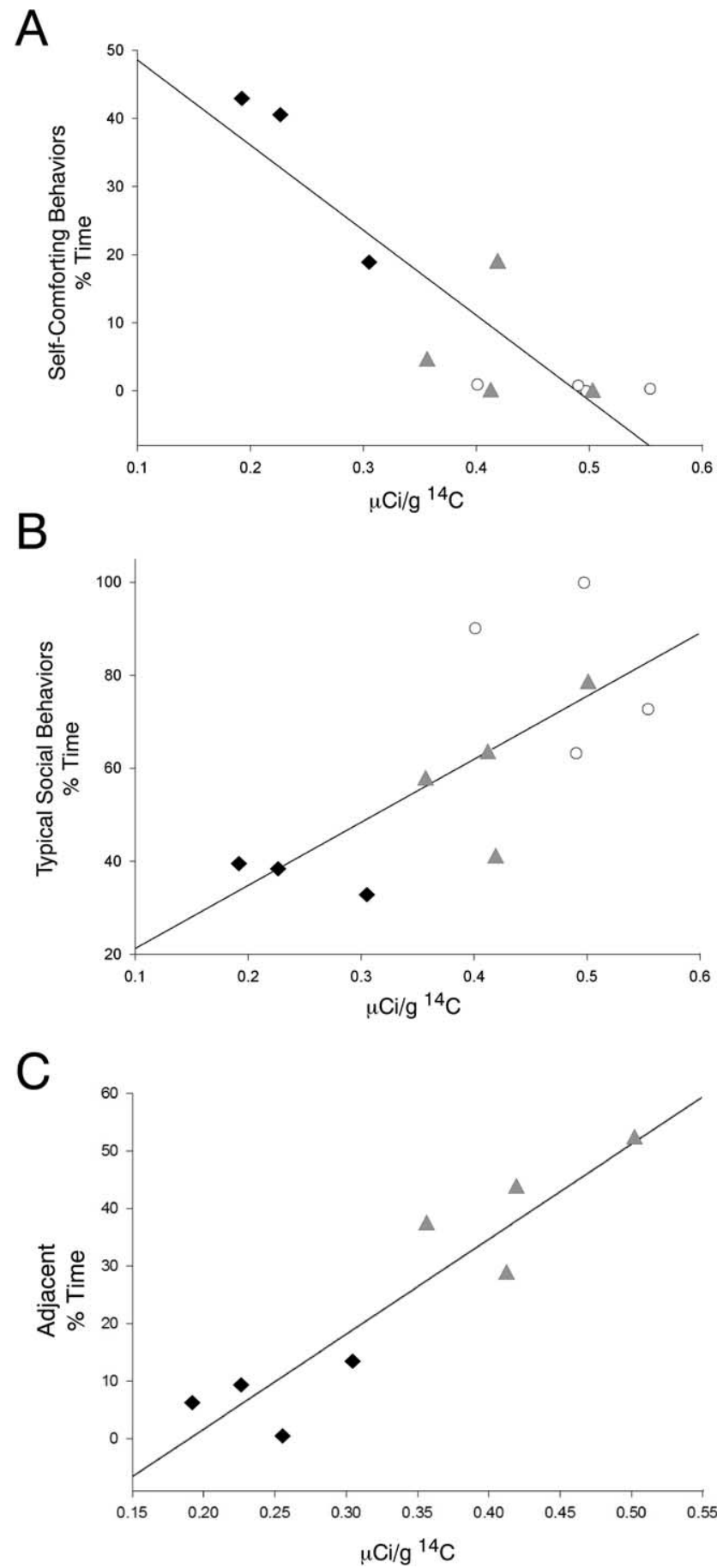

Figure 5. Gene-behavior correlations. Black filled diamonds represent individual 1 week separated monkeys, gray filled triangles represent individual 1 month separated monkeys, and white open circles represent individual maternally reared monkeys. $A$, Level of self-comforting behavior during the third month of life is significantly correlated with GUCY1A3 mRNA levels in the amygdala $(r=-0.878 ; p<0.001)$. B, Level of typical social behavior during the third month of life is significantly correlated with GUCY1A3 mRNA levels in the amygdala $(r=0.708$; $p=0.015)$. C, Level of social-comforting in the acute separation period is correlated with GUCY1A3 mRNA level at 3 months of age $(r=0.923 ; p=0.001)$.

deficits that are critical for the development of the observed behaviors.

Importantly, in our study, the adult level of GUCY1A3 gene expression was already in place by 1 week of age, suggesting that the downregulation of GUCY1A3 resulted from a decreased ex- pression from normal levels and was not a consequence of a failed developmental increase over the time window studied. Thus, given the level of the reduction in GUCY1A3 expression ( 50\%) and the grossly normal appearance of Nissl sections regardless of separation condition, we favor the possibility that normally expressing cells decrease their expression over the alternate possibility that subsets of cells that express GUCY1A3 mRNA are eliminated. In our study, specific labeling by antisense riboprobes was located around and over lightly counterstained, large cells in all subnuclei examined. By virtue of their morphology, these neurons are likely to be pyramidal-like projection neurons (Pare et al., 1995; Faber et al., 2001), although we cannot exclude the possibility that a subset of interneurons also produce the GUCY1A3 transcript.

Although GUCY1A3 is most likely expressed in excitatory cells, its role in the activity of the cell remains uncertain. Indeed, NO has been shown to have excitatory, inhibitory, and modulatory effects depending on where and how it is studied (Dawson and Snyder, 1994; Mothet et al., 1996). Thus, the GUCY1A3 transcript downregulation in our studies could have arisen via one of two distinct mechanisms. First, maternal separation may have led to a dysregulation of the NO signaling pathway in the amygdala as a direct result of a primary GUCY1A3 transcript downregulation. Alternatively, GUCY1A3 downregulation may represent an insufficient adaptational molecular response directed toward the downregulation of pathologically increased NO signaling in the maternally separated monkeys. Future studies that perturb this pathway in the monkey amygdala via pharmacological or molecular methods may differentiate between these possibilities.

Regardless of the exact mechanism by which the GUCY1A3 downregulation arose, all our findings suggest that the observed correlation between GUCY1A3 expression and salient behaviors in the social group deserves further attention. These findings are highly suggestive that GUCY1A3 acts as a putative modifier of socioemotional behaviors directed by the amygdala.

\section{References}

Agid O, Shapira B, Zislin J, Ritsner M, Hanin B, Murad H, Troudart T, Bloch M, Heresco-Levy U, Lerer B (1999) Environment and vulnerability to major psychiatric illness: a case control study of early parental loss in major depression, bipolar disorder and schizophrenia. Mol Psychiatry 4:163-172.

Ahern GP, Klyachko VA, Jackson MB (2002) cGMP and S-nitrosylation: two routes for modulation of neuronal excitability by NO. Trends Neurosci 25:510-517.

Amaral DG (2003) The amygdala, social behavior, and danger detection. Ann NY Acad Sci 1000:337-347.

Baron-Cohen S, Ring HA, Bullmore ET, Wheelwright S, Ashwin C, Williams SC (2000) The amygdala theory of autism. Neurosci Biobehav Rev 24:355-364.

Bauman MD, Lavenex P, Mason WA, Capitanio JP, Amaral DG (2004) The development of social behavior following neonatal amygdala lesions in rhesus monkeys. J Cogn Neurosci 16:1388-1411.

Brothers L (1990) The social brain: a project for integrating primate behavior and neurophysiology in a new domain. Concepts Neurosci 1:27-51.

Cameron JL (2001) Critical periods for social attachment: deprivation and neural systems in rhesus monkeys. Soc Res Child Development Abstr 2-054.

Chien WL, Liang KC, Teng CM, Kuo SC, Lee FY, Fu WM (2003) Enhancement of long-term potentiation by a potent nitric oxide-guanylyl cyclase activator, 3-(5-hydroxymethyl-2-furyl)-1-benzyl-indazole. Mol Pharmacol 63:1322-1328.

Chien WL, Liang KC, Teng CM, Kuo SC, Lee FY, Fu WM (2005) Enhancement of learning behaviour by a potent nitric oxide-guanylate cyclase activator YC-1. Eur J Neurosci 21:1679-1688.

Chisholm K (1998) A three year follow-up of attachment and indiscriminate friendliness in children adopted from Romanian orphanages. Child Dev 69:1092-1106. 
Dawson TM, Snyder SH (1994) Gases as biological messengers: nitric oxide and carbon monoxide in the brain. J Neurosci 14:5147-5159.

Drevets WC (1999) Prefrontal cortical-amygdalar metabolism in major depression. Ann NY Acad Sci 877:614-637.

Drevets WC, Bogers W, Raichle ME (2002) Functional anatomical correlates of antidepressant drug treatment assessed using PET measures of regional glucose metabolism. Eur Neuropsychopharmacol 12:527-544.

Emery NJ, Capitanio JP, Mason WA, Machado CJ, Mendoza SP, Amaral DG (2001) The effects of bilateral lesions of the amygdala on dyadic social interactions in rhesus monkeys (Macaca mulatta). Behav Neurosci 115:515-544.

Etkin A, Klemenhagen KC, Dudman JT, Rogan MT, Hen R, Kandel ER, Hirsch J (2004) Individual differences in trait anxiety predict the response of the basolateral amygdala to unconsciously processed fearful faces. Neuron 44:1043-1055.

Faber ES, Callister RJ, Sah P (2001) Morphological and electrophysiological properties of principal neurons in the rat lateral amygdala in vitro. J Neurophysiol 85:714-723.

Furuyama T, Inagaki S, Takagi H (1993) Localizations of alpha 1 and beta 1 subunits of soluble guanylate cyclase in the rat brain. Brain Res Mol Brain Res 20:335-344.

Gonzalez-Burgos G, Barrionuevo G, Lewis DA (2000) Horizontal synaptic connections in monkey prefrontal cortex: an in vitro electrophysiological study. Cereb Cortex 10:82-92.

Gonzalez-Burgos G, Zaitsev AV, Povysheva AV, Krimer LS, Barrionuevo G, Lewis DA (2005) Functional maturation of excitatory synapses during postnatal development of the primate prefontal cortex. Soc Neurosci Abstr 31:26.14.

Harlow HF, Suomi SJ (1971) Production of depressive behaviors in young monkeys. J Autism Child Schizophr 1:246-255.

Harlow HF, Suomi SJ (1974) Induced depression in monkeys. Behav Biol 12:273-296.

Harlow HF, Zimmermann RR (1959) Affectional responses in the infant monkey; orphaned baby monkeys develop a strong and persistent attachment to inanimate surrogate mothers. Science 130:421-432.

Hatzidimitriou G, McCann UD, Ricaurte GA (1999) Altered serotonin innervation patterns in the forebrain of monkeys treated with $( \pm) 3,4-$ methylenedioxymethamphetamine seven years previously: factors influencing abnormal recovery. J Neurosci 19:5096-5107.

Hawkins RD, Son H, Arancio O (1998) Nitric oxide as a retrograde messenger during long-term potentiation in hippocampus. Prog Brain Res 118:155-172.

He Y, Yu W, Baas PW (2002) Microtubule reconfiguration during axonal retraction induced by nitric oxide. J Neurosci 22:5982-5991.

Hess DT, Patterson SI, Smith DS, Skene JH (1993) Neuronal growth cone collapse and inhibition of protein fatty acylation by nitric oxide. Nature 366:562-565.

Hindley S, Juurlink BH, Gysbers JW, Middlemiss PJ, Herman MA, Rathbone MP (1997) Nitric oxide donors enhance neurotrophin-induced neurite outgrowth through a cGMP-dependent mechanism. J Neurosci Res 47:427-439.

Holscher C, McGlinchey L, Anwyl R, Rowan MJ (1996) 7-Nitro indazole, a selective neuronal nitric oxide synthase inhibitor in vivo, impairs spatial learning in the rat. Learn Mem 2:267-278.

Irizarry RA, Bolstad BM, Collin F, Cope LM, Hobbs B, Speed TP (2003) Summaries of Affymetrix GeneChip probe level data. Nucleic Acids Res 31:e15.

Lorberbaum JP, Kose S, Johnson MR, Arana GW, Sullivan LK, Hamner MB, Ballenger JC, Lydiard RB, Brodrick PS, Bohning DE, George MS (2004) Neural correlates of speech anticipatory anxiety in generalized social phobia. NeuroReport 15:2701-2705.

McCormick K, Gualano MF, Kerr D, Rockcastle N, Cameron JL (2005) Social bond disruption in early life has behavioral consequences which remain evident through puberty. Soc Neurosci Abstr 31:873.9.

McDonald AJ, Augustine JR (1993) Localization of GABA-like immunoreactivity in the monkey amygdala. Neuroscience 52:281-294.

McDonald AJ, Payne DR, Mascagni F (1993) Identification of putative nitric oxide producing neurons in the rat amygdala using NADPHdiaphorase histochemistry. Neuroscience 52:97-106.

Mireault GC, Bond LA (1992) Parental death in childhood: perceived vulnerability, and adult depression and anxiety. Am J Orthopsychiatry 62:517-524.

Mirnics K, Middleton FA, Stanwood GD, Lewis DA, Levitt P (2001) Disease-specific changes in regulator of G-protein signaling 4 (RGS4) expression in schizophrenia. Mol Psychiatry 6:293-301.
Monzon ME, Varas MM, De Barioglio SR (2001) Anxiogenesis induced by nitric oxide synthase inhibition and anxiolytic effect of melaninconcentrating hormone $(\mathrm{MCH})$ in rat brain. Peptides 22:1043-1047.

Mothet JP, Fossier P, Schirar A, Tauc L, Baux G (1996) Opposite effects of nitric oxide on identified inhibitory and excitatory cholinergic synapses of Aplysia californica. Physiol Res 45:177-183.

Nelson CA, Bloom FE, Cameron JL, Amaral D, Dahl RE, Pine D (2002) An integrative, multidisciplinary approach to the study of brain-behavior relations in the context of typical and atypical development. Dev Psychopathol 14:499-520.

O'Connor TG, Rutter M (2000) Attachment disorder behavior following early severe deprivation: extension and longitudinal follow-up. English and Romanian Adoptees Study Team. J Am Acad Child Adolesc Psychiatry 39:703-712.

Pare D, Pape HC, Dong J (1995) Bursting and oscillating neurons of the cat basolateral amygdaloid complex in vivo: electrophysiological properties and morphological features. J Neurophysiol 74:1179-1191.

Paton JJ, Belova MA, Morrison SE, Salzman CD (2006) The primate amygdala represents the positive and negative value of visual stimuli during learning. Nature 439:865-870.

Petrovich GD, Holland PC, Gallagher M (2005) Amygdalar and prefrontal pathways to the lateral hypothalamus are activated by a learned cue that stimulates eating. J Neurosci 25:8295-8302.

Rialas CM, Nomizu M, Patterson M, Kleinman HK, Weston CA, Weeks BS (2000) Nitric oxide mediates laminin-induced neurite outgrowth in PC12 cells. Exp Cell Res 260:268-276.

Rodman HR, Consuelos MJ (1994) Cortical projections to anterior inferior temporal cortex in infant macaque monkeys. Vis Neurosci 11:119-133.

Russwurm M, Koesling D (2004) Guanylyl cyclase: NO hits its target. Biochem Soc Symp (71):51-63.

Rutter M, O'Connor TG (2004) Are there biological programming effects for psychological development? Findings from a study of Romanian adoptees. Dev Psychol 40:81-94.

Rutter M, Andersen-Wood L, Beckett C, Bredenkamp D, Castle J, Groothues C, Kreppner J, Keaveney L, Lord C, O'Connor TG (1999) Quasi-autistic patterns following severe early global privation. English and Romanian Adoptees (ERA) Study Team. J Child Psychol Psychiatry 40:537-549.

Smigrodzki R, Levitt P (1996) The alpha 1 subunit of soluble guanylyl cyclase is expressed prenatally in the rat brain. Brain Res Dev Brain Res 97:226-234.

Son H, Lu YF, Zhuo M, Arancio O, Kandel ER, Hawkins RD (1998) The specific role of cGMP in hippocampal LTP. Learn Mem 5:231-245.

Storey JD, Tibshirani R (2003) Statistical significance for genomewide studies. Proc Natl Acad Sci USA 100:9440-9445.

Suomi SJ, Eisele CD, Grady SA, Harlow HF (1975) Depressive behavior in adult monkeys following separation from family environment. J Abnorm Psychol 84:576-578.

Volk DW, Austin MC, Pierri JN, Sampson AR, Lewis DA (2000) Decreased glutamic acid decarboxylase67 messenger RNA expression in a subset of prefrontal cortical gamma-aminobutyric acid neurons in subjects with schizophrenia. Arch Gen Psychiatry 57:237-245.

Volke V, Koks S, Vasar E, Bourin M, Bradwejn J, Mannisto PT (1995) Inhibition of nitric oxide synthase causes anxiolytic-like behaviour in an elevated plus-maze. NeuroReport 6:1413-1416.

Volke V, Soosaar A, Koks S, Bourin M, Mannisto PT, Vasar E (1997) 7-Nitroindazole, a nitric oxide synthase inhibitor, has anxiolytic-like properties in exploratory models of anxiety. Psychopharmacology (Berl) 131:399-405.

Webster MJ, Ungerleider LG, Bachevalier J (1991) Connections of inferior temporal areas TE and TEO with medial temporal-lobe structures in infant and adult monkeys. J Neurosci 11:1095-1116.

Weindruch R, Kayo T, Lee CK, Prolla TA (2002) Effects of caloric restriction on gene expression. Nestle Nutr Workshop Ser Clin Perform Programme 6:17-28; discussion 28-32.

Werner C, Raivich G, Cowen M, Strekalova T, Sillaber I, Buters JT, Spanagel R, Hofmann F (2004) Importance of NO/cGMP signalling via cGMPdependent protein kinase II for controlling emotionality and neurobehavioural effects of alcohol. Eur J Neurosci 20:3498-3506.

Zabel U, Weeger M, La M, Schmidt HH (1998) Human soluble guanylate cyclase: functional expression and revised isoenzyme family. Biochem J 335:51-57. 